\title{
Contact between representative rough surfaces
}

\author{
Vladislav A. Yastrebov ${ }^{1,2 *}$, Guillaume Anciaux ${ }^{2 \dagger}$, Jean-François Molinari2 ${ }^{2 \ddagger}$ \\ ${ }^{1}$ Centre des Matériaux, MINES ParisTech, CNRS UMR 7633, \\ BP 87, 91003, Evry, France \\ ${ }^{2}$ Computational Solid Mechanics Laboratory (LSMS, IIC-ENAC, IMX-STI), \\ Ecole Polytechnique Fédérale de Lausanne (EPFL), \\ Station 18, 1015, Lausanne, Switzerland
}

October 31, 2018

\begin{abstract}
A numerical analysis of mechanical frictionless contact between rough selfaffine elastic manifolds was carried out. It is shown that the lower cutoff wavenumber in surface spectra is a key parameter controlling the representativity of the numerical model. Using this notion we demonstrate that for representative surfaces the evolution of the real contact area with load is universal and independent of the Hurst roughness exponent. By introducing a universal law containing three constants, we extend the study of this evolution beyond the limit of infinitesimal area fractions.
\end{abstract}

\section{Introduction}

Real surfaces are self-affine [1, 2] and the surface heights are distributed normally [3]. Due to this roughness the real contact area $A$ is often only a

*vladislav.yastrebov@mines-paristech.fr

†'guillaume.anciaux@epfl.ch

†jean-francois.molinari@epfl.ch 
small fraction of the nominal or apparent contact area $A_{0}$. The real contact area fraction $A / A_{0}$ and its evolution determines the contact resistivity, friction and the transfer of energy (heat and electric charge) through the contact interface. Thus understanding the contact-area evolution has profound implications in various fundamental (e.g., origin of friction) and engineering studies (e.g., electro-mechanical contact, tire-road interaction). From early experiments [4] and analytical theories [3, 5], it has been considered as an established knowledge that $A$ evolves linearly with the normal load $F$ at relatively small fractions of contact. It was confirmed by numerical simulations of normal frictionless elastic contact between rough surfaces [6], which made considerable progress since then. Nowadays, the most advanced computations exploit fine surface discretizations $(2048 \times 2048$ [7, 8, 9, 10], $4096 \times 4096$ [11]) to extract statistically meaningful results valid even for small fractions of contact. Many of the numerical investigations are based on synthesized surfaces preserving the aspect of self-affinity. In early studies [6, 12] the artificial rough surfaces obeyed fractality which scaled down to the domain discretization [13]. Later it was realized that the surface has to be smooth enough [7, 14] to represent correctly the mechanics of contact and to obtain a reliable estimation of the contact area growth comparable with analytical theories. This smoothness implies a sharp decay of the power spectrum density for wavelengths smaller than short cutoff wavelength $\lambda_{s}$. On the other hand, to the best of our knowledge, there are no consistent study on the influence of long cutoff wavelength $\lambda_{l}$ on the response of rough surfaces. Despite remarks made in [15] on the importance of $\lambda_{l}$ for mechanics of rough contact, in almost all investigations of the real-contact-area evolution [6, 17, 10, 16] $\lambda_{l}$ was limited by the size of the specimen $\lambda_{l}=L$. In this article we show that $\lambda_{l}$ plays a crucial role in numerically precise theory of rough contact, because it controls the representativity of the simulated system.

In the limit of infinitesimal contact, analytical theories predict that the real contact area $A$ evolves proportionally to the normal force $F$ with coefficient $\kappa$ normalized by the root mean squared slope of the surface $\sqrt{\left\langle|\nabla h|^{2}\right\rangle}$ and the effective Young's modulus $E^{*}$ [17], which gives

$$
A=\kappa / \sqrt{\left\langle|\nabla h|^{2}\right\rangle} F / E^{*}
$$

According to asperity-based [3] models (Bush, Gibson, Thomas (BGT) [5], Greenwood [18], Nayak-Thomas [19]) $\kappa=\sqrt{2 \pi}$ but Eq. (11) is valid only in an asymptotic limit for infinitesimal fractions of the contact area $A / A_{0} \rightarrow 0$. 


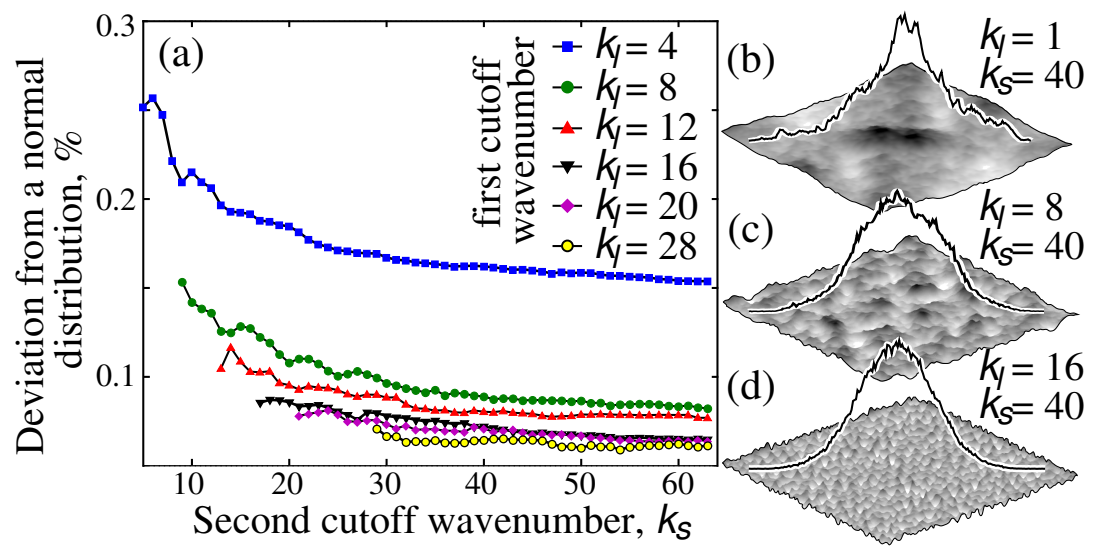

FIG. 1: Representativity of a rough self-affine surface can be estimated according to the normality of heights distribution. Here, the criterion of proximity is an error between the distribution of surface's heights and the associated normal distribution; (a) this error is strongly dependent on the cutoff wavenumbers $k_{s}$ and $k_{l}$. Every point on the graph represents an error averaged over 30 statistically equivalent surfaces. Examples of rough surfaces and the corresponding heights distributions for different cutoffs $k_{s}=40$ and (b) $k_{l}=1$, (c) $k_{l}=4$, (d) $k_{l}=16$ are also depicted. 
The convergence of $\kappa$ with decreasing fraction $A / A_{0}$ is slow and depends on the bandwidth parameter $\alpha$ introduced in [20] as $\alpha=m_{0} m_{4} / m_{2}^{2}$, where

$m_{i}=\int_{k_{l}}^{k_{s}} k^{i} C(k) d k$ is the $i$-th moment of the power spectrum density $C(k)$ of the surface. For instance, for $\alpha=10, \kappa$ is overestimated by about $12 \%$ for $A / A_{0}=10^{-5}$ and this error reduces only to $4 \%$ when $A / A_{0}=10^{-10}$ [18]. Consequently, using a complete numerical model it is not possible to demonstrate the linearity predicted by Eq. (1) nor to approach its asymptotic limit. It is also worth noting that in forementioned models the evolution of the real contact area is strictly nonlinear for realistic fractions of contact [21, 22]. Moreover, this evolution does not depend on the Hurst roughness exponent $H$ but only on $\alpha$ [21]. A competing theory was proposed by Persson [23, 24, it also predicts a linear contact area evolution for small contact fractions, however, the obtained coefficient of proportionality $\kappa=\sqrt{8 / \pi}$ is significantly smaller than in the asperity-based models. As in the latter models, the evolution of the contact area does not depend on the Hurst exponent. Numerical studies [6, 7, 10, 16] demonstrated an approximately linear evolution of the contact area with load. But in contrast to the analytical models, $\kappa$ was shown to depend on the Hurst exponent and to be confined between the asymptotic limits of the BGT [5] and Persson [23, 24] theories. In this article, by introducing the notion of surface representativity, we obtain qualitatively new numerical results, which we believe correctly represent the mechanical response of realistic rough surfaces. Moreover, they appeal to a broad interpretation of the data not restricted to a single proportionality coefficient between contact area and load for infinitesimal contact fractions.

\section{Representativity of rough surfaces}

A meaningful numerical simulation of rough contact has to be carried out on a representative self-affine surface element (RSSE) (e.g. 25]) either generated numerically or chosen from experimental measurements. The reason to use generated rough surfaces is that they may be obtained for any cutoff wavelengths and Hurst exponent $H$. On the one hand, the RSSE has to be large enough to obtain a similar response for different realizations of statistically equivalent surfaces. On the other hand, it has to be as small as possible to retain a numerically solvable contact problem. So the RSSE is defined according to the permitted error between mechanical responses of surfaces of different sizes, e.g. $L$ and $2 L$. This mechanical representativity can be 


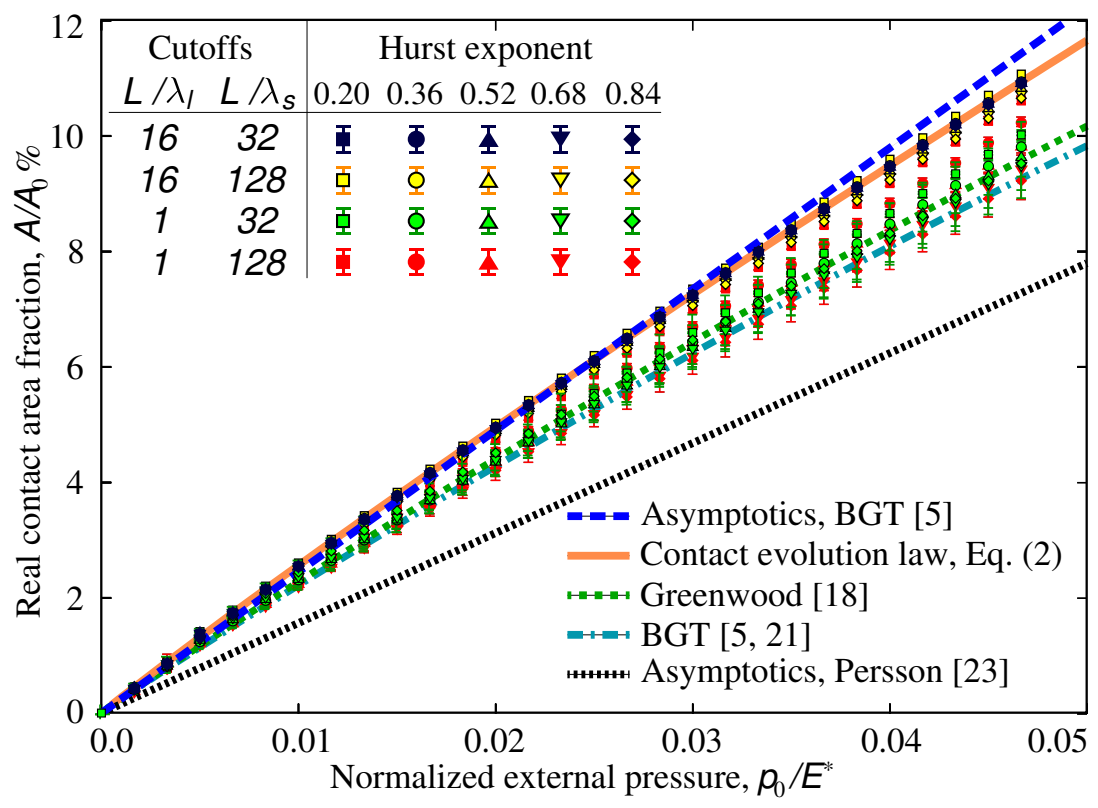

FIG. 2: Evolution of the real contact area fraction $A / A_{0}$ with normalized external load $p_{0} / E^{*}$ are plotted for different cutoff wavelengths and Hurst exponents; the rms slope is kept constant for all surfaces $\sqrt{\left\langle|\nabla h|^{2}\right\rangle}=0.1$. The results for representative surfaces $\left(\lambda_{l}=L / 16\right)$ are in a good agreement with the asymptotic limit of the Bush, Gibson, Thomas (BGT) theory [5] and are perfectly fitted by the suggested contact evolution law Eq. (2) $(\kappa=1.145 \sqrt{2 \pi}, \mu=$ $0.55, \beta=0.21)$. Moreover, if the surface is sufficiently smooth $\lambda_{s}=L / 32$ we do not observe any dependence of results on the Hurst exponent. The evolution of the real contact area predicted by Greenwood [18] and BGT [5] theories for spectrum bandwidth $m_{0} m_{4} / m_{2}^{2}=2$ (data from [21]) lie in the confidence interval of the results obtained for non-representative surfaces $\left(\lambda_{l}=L\right)$ with high Hurst exponents $H \gtrsim 0.5$. Asymptotic limit of Persson theory [23, 24] lies below all obtained results. 
linked to the geometrical representativity, which we define as the proximity of the surface heights distribution to a normal distribution [14]. In particular, this proximity is important in the range of maximal heights corresponding to the only zones which come in contact at small loads. To analyze how the representativity depends on the cutoffs, for each pair of cutoff wavenumbers $k_{s}=L / \lambda_{s}$ and $k_{l}=L / \lambda_{l}$ we generate 30 statistically equivalent surfaces using FFT filtering algorithm [26]. The size of surfaces is $L=1$, the discretization spacing is $\Delta L=1 / 1024$ and $H=0.8$. Fig. 1 represents the average $L 2$ error between the heights distribution function of the generated surfaces and the corresponding normal distributions $G(h, \bar{h}, \sigma)$ for different cutoffs (see appendix for details). The mean value $\bar{h}$ and the standard devia-

tion $\sigma=\sqrt{\left\langle(\bar{h}-h)^{2}\right\rangle}$ for the normal distribution $G$ are computed according to the generated surface. It follows from this figure that a generated surface becomes more and more representative, when the first cutoff wavenumber $k_{l}$ increases up to $8-16$, whereas the variation of $k_{s}$ has a weaker effect on the surface representativity if the spectrum is sufficiently rich $k_{s} \gg k_{l}$. The choice $\lambda_{l}=L$ leads to a surface with a mechanical response which varies considerably from one surface realization to another. The fact that in average this response does not correspond to the response of a bigger rough surface with similar random properties is of crucial importance for the study of rough contact. The key reasons for this discrepancy are the long-range interactions $(1 / r)$ between contacting asperities and inevitable boundary conditions on lateral sides of the specimen (periodic, symmetric, free). Accordingly, the response of any heterogeneous system with random properties and long-range interactions should be studied on a representative system element.

\section{Smoothness of rough surfaces}

Besides the long cutoff wavelength, in generated or experimental surfaces there is a limitation connected with their inevitable discreteness, either due to a numerical resolution scheme and/or experimental measurements. In real self-affine surfaces the power spectrum density decays as a power-law of the wavenumber $k, C(k) \sim k^{-2(1+H)}$. This law may be preserved down to wavelengths comparable to atomic spacings [2], where the continuum contact mechanics is not valid anymore [27]. To remain in the continuum framework and to capture accurately the mechanics, we introduce a short cutoff wavelength $\lambda_{s}>\Delta L$, which ensures the smoothness of surfaces at a certain magnifica- 


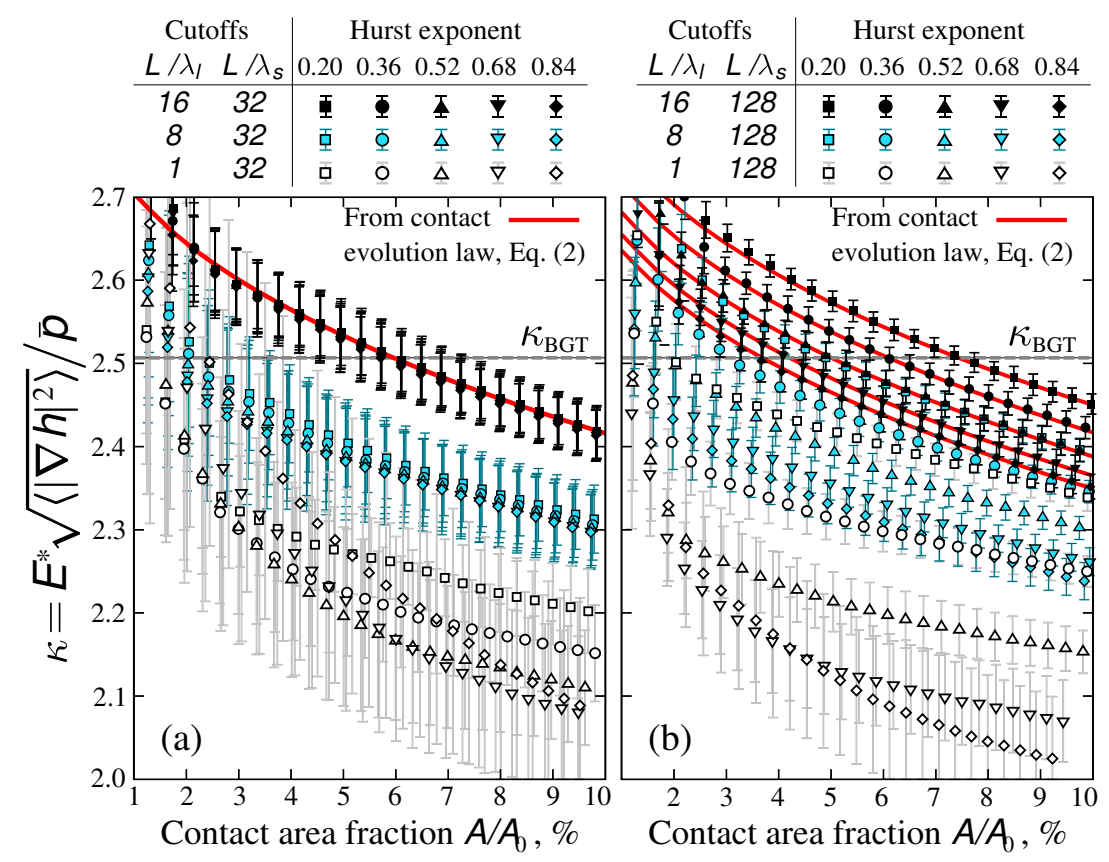

FIG. 3: Evolution of $\kappa$ with the real contact area fraction $A / A_{0}$ is depicted for different Hurst roughness exponents and cutoff wavelengths $\lambda_{l}=L, L / 8, L / 16$, a) $\lambda_{s}=L / 32$, b) $\lambda_{s}=L / 128$; all results for representative surfaces $\lambda_{l}=L / 16$ are fitted by the contact evolution law Eq. (2) with $\kappa=1.145 \sqrt{2 \pi}, \mu=0.55, \beta=$ 0.21 for (a) $\lambda_{l}=L / 16, \lambda_{s}=L / 32$; with $\kappa=1.145 \sqrt{2 \pi}, \mu=0.56$ and $\beta \in$ $[1.120,1.172]$ for (b) $\lambda_{l}=L / 16, \lambda_{s}=L / 128$. For comparison, the asymptotic limit $\kappa_{\mathrm{BGT}}=\sqrt{2 \pi}$ from Bush, Gibson, Thomas theory [5] is plotted, while the asymptotic limit of Persson theory [23, 24] $\kappa_{\mathrm{P}}=\sqrt{8 / \pi} \approx 1.60$ is out of the plot range. 
tion as, for example, in [7]. The inherent cutoff $\lambda_{s}=\Delta L$ used, for example, in [6, 12] results in a "one node - one asperity" approach, which does not allow to correctly reproduce the local change of the contact force with separation [14] nor to estimate a realistic growth of localized contact zones but only the growth of their number. Considering smooth-rough-surfaces with a "truncated self-affinity" $\lambda_{s} \gg \Delta L$ eliminates these shortcomings.

\section{Description of simulations}

Using the algorithm [26] we generate 12 statistically equivalent surfaces for each pair of cutoff wavelengths $L / \lambda_{l}=1,2,8,16, L / \lambda_{s}=32,64,128,256,512$ and different Hurst roughness exponents $H=0.2,0.36,0.52,0.68,0.84$. For all the surfaces the constant root mean squared slope $\sqrt{\left\langle|\nabla h|^{2}\right\rangle}=0.1$ is preserved; as before $L=1$ and $\Delta L=1 / 1024$. It is important to note that, in contrast to geometrical estimations of $\sqrt{\left\langle|\nabla h|^{2}\right\rangle}$ [6], which depend on the surface discretization and thus may underestimate significantly the real value, we evaluate the rms slope according to the surface power spectrum [20]. To solve the contact problem between a periodic rigid rough surface and a deformable flat half-space we use the spectral based boundary element method [28] with some minor improvements [29]. This method allows to solve accurately the equations of continuum mechanics under contact constraints and, in contrast to asperity-based models, takes into account all underlying mechanics: complex shapes of asperities, junction of contact zones associated with different asperities and long-range deformation of the elastic half-space in response to contact forces. For each simulation the contact area fraction reaches $\approx 10 \%$ under the external pressure linearly increasing up to $p_{0} / E^{*}=0.05$ within 30 increments. In Fig. 2 we compare the area-force curves of our numerical results and several analytical theories, which were computed and summarized in [21]. To demonstrate better the nonlinearity of the area evolution for surfaces with different cutoffs and Hurst exponents, we present in Fig. 3 the proportionality coefficient $\kappa$ expressed from Eq. 1 as $\kappa=E^{*} \sqrt{\left\langle|\nabla h|^{2}\right\rangle} / \bar{p}$, where $\bar{p}=F / A$ is the mean contact pressure. 


\section{Results}

The value of $\kappa$ for the observed range (up to $10 \%$ of the contact fraction) is a decreasing function of the area. For non-representative $\lambda_{l}<L / 16$ or too rough $\lambda_{s}<32 \Delta L$ surfaces, there is a clear tendency (see Fig. 3, b): a higher $H$ results in a smaller $\kappa$. This dependence of the results on the Hurst exponent corresponds to all up to date numerical investigations of the $\kappa$ constant [6, 17, 16, 10]. However, we argue that these results are strongly affected by non-representativity and an excessive roughness of exploited surfaces. In contrast to these results, if the mechanics of contact is well resolved $\left(\lambda_{s} \gg \Delta L\right)$ and the surface is representative $\left(\lambda_{l} \gtrsim L / 16\right)$ the real contact area evolution does not depend anymore on the Hurst exponent (see dark symbols in Fig. 3 , a). This result is in a good agreement with analytical theories and has never been obtained before in complete numerical models. Reducing $\lambda_{l}$ reduces the data scatter, which is one of criteria of mechanical representativity. The data scatter increases with increasing $H$, thus, the smoother the surface, the bigger RSSE is needed to capture an average mechanical behavior. The rise in $\kappa$ with decreasing $\lambda_{l}$ can be interpreted in terms of Persson theory [23, 24]: reducing $\lambda_{l}$ is equivalent to decreasing magnification which rises $\kappa$. Note that our results for $\kappa$ are not confined between the asymptotic limits of the BGT [5] $\kappa_{\mathrm{BGT}}=\sqrt{2 \pi}$ and Persson theories $\kappa_{\mathrm{P}}=\sqrt{8 / \pi}$ [23], as was generally observed by other authors [6, 7, 16, 10].

\section{Phenomenological Contact Evolution Law}

In light of these results, we propose a new phenomenological contact evolution law which is based on our observations of the change in the mean contact pressure $\bar{p}=F / A$ with respect to the applied pressure $p_{0}=F / A_{0}$ depicted in Fig. 4. For considered interval of contact areas $A / A_{0} \in[0.01,0.11]$ the decay of $\partial \bar{p} / \partial p_{0}$ can be approximated by power-law $\partial \bar{p} / \partial p_{0}=\beta\left(A_{0} / A\right)^{\mu}$, $0<\mu<1$. Therefore, we obtain (for details see appendix the evolution of the real contact area as

$$
A / A_{0}=\left(\beta+\left[\kappa p_{0} /\left(\sqrt{\left\langle|\nabla h|^{2}\right\rangle} E^{*}\right)\right]^{\mu-1}\right)^{1 /(\mu-1)}
$$

where $\kappa$ is still a proportionality constant between the area and normalized force for infinitesimal pressures, two other constants $\mu$ and $\beta$ can be easily 
and uniquely found from the data analysis. For converged results $\lambda_{l}=L / 16$, $\lambda_{s}=L / 32$ by fitting the normalized inverse mean pressure (Fig. (3) we found $\kappa=(1.145 \pm 0.002) \sqrt{2 \pi}$, which is about $15 \%$ higher than the asymptotic limit of the BGT theory [5]. From Fig. 4 we uniquely obtain $\mu=0.55 \pm 0.02$, $\beta=0.210 \pm 0.005$.

\section{Conclusions}

To study the growth of the real contact area between rough self-affine manifolds we obtained statistically meaningful results for different cutoff wavelengths and Hurst exponents. We demonstrated that the real contact area evolves nonlinearly with applied force even for reasonably small contact fractions $A / A_{0}<0.1$. Note that an almost linear evolution was observed for rough contact between elasto-plastic materials [24, 12, 14], however, in this case the Johnson's assumption [17] on replacing two deformable solids by one solid with effective elasto-plastic properties and superposed roughnesses is not verified and a full simulation of two deformable solids is required.

In conclusion, we state that to obtain realistic results in rough contact analysis, one needs to construct representative surfaces. This representativity necessarily requires the long wavelength cutoff to be significantly smaller than the specimen size $\lambda_{l} \gtrsim L / 16$. The Hurst exponent does not change the evolution of the real contact area with load if both the representativity and the smoothness of surfaces are maintained. To describe the evolution of the real contact area we proposed a new phenomenological law which describes well our results for moderate pressures and reduces to the classical BGT law [5] in the limit of infinitesimal contact fractions. However, further work is required to verify the universality of this law. In perspective we aim

to study the pressure distribution and the evolution of the contact area up to the full contact, for which the numerical results should be in a better agreement with Persson theory [23, 24]. An important question, which we could not answer in our study, is how the bandwidth parameter influences the mechanical behavior of rough surfaces. To address it, one needs to carry out numerical simulations on a considerably finer discretization of surfaces than was reported here. We hope that our results will motivate new simulations based on the notion of representativity and taking into account friction, viscoplasticity, adhesion and surface energy.

The financial support from the European Research Council (ERCstg 


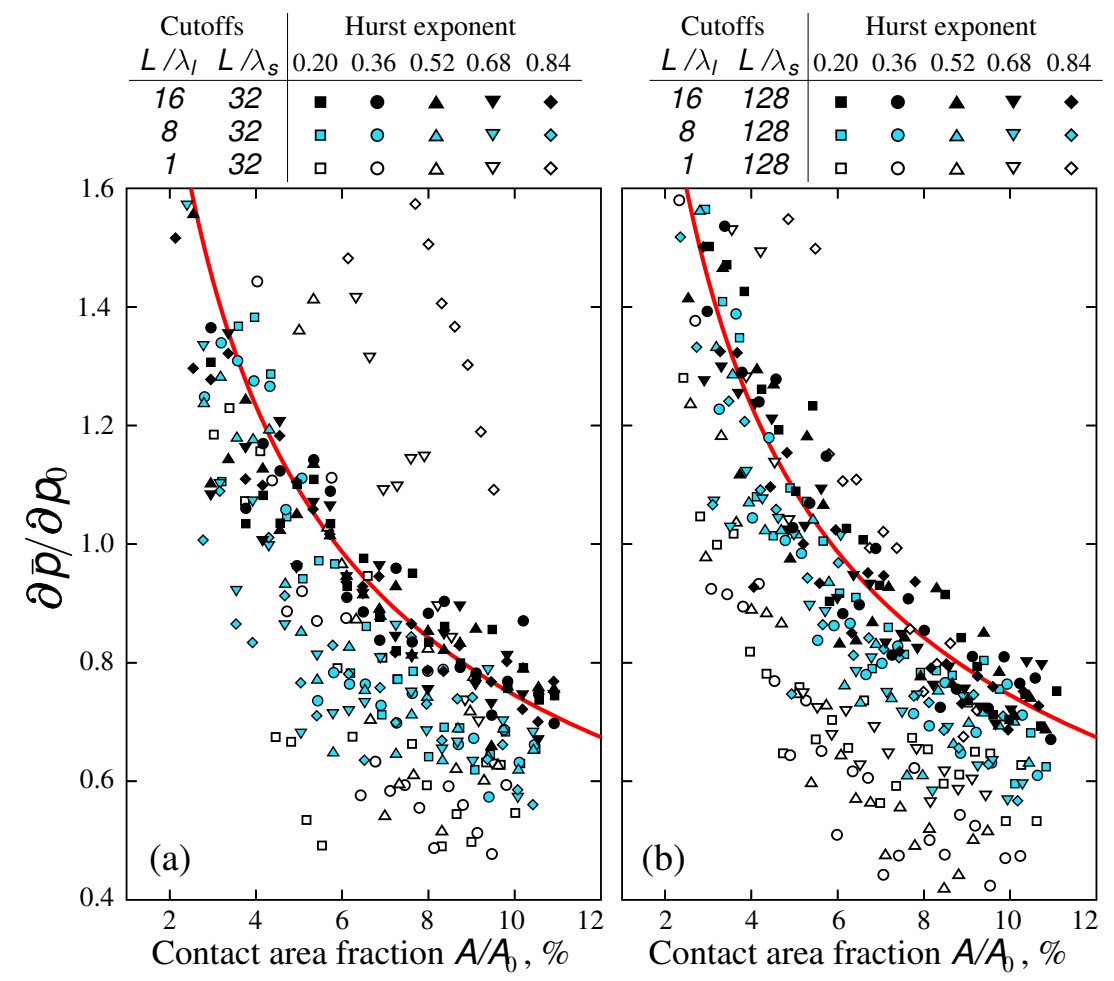

FIG. 4: The mean pressure derivative with respect to the external pressure $\partial \bar{p} / \partial p_{0}$ decreases nonlinearly with increasing fraction of the real contact area $A / A_{0}$. We plot its evolution for different Hurst roughness exponents and cutoff wavelengths $\lambda_{l}=L, L / 8, L / 16$ and (a) $\lambda_{s}=L / 32$, (b) $\lambda_{s}=L / 128$. For representative surfaces the results can be roughly approximated by a power law $\partial \bar{p} / \partial p_{0}=\beta\left(A_{0} / A\right)^{\mu}$; solid line corresponds to parameters $\beta=0.21, \mu=0.55$, which fit well our results for representative $\lambda_{l}=L / 16$ smooth surfaces. 
UFO-240332) is greatly acknowledged.

\section{Appendix: representativity of rough sur- faces}

The generation of realistic surfaces is the basis for a numerical analysis of the contact interaction between solids. It is widely acknowledged that real surfaces obey the property of self-affinity [17, 2] over many spatial scales. Self-affine surfaces can be described by a power spectrum density (PSD) $C(k)$ following a power law of a wavenumber $k=L / \lambda$

$$
C(k) \sim k^{-2(H+1)}
$$

where $H$ is the so-called Hurst roughness exponent $H \in(0,1)$. In reality the PSD may be considered confined between two cutoff wavenumbers: $k_{s}$ and $k_{l}$ corresponding to short and long wavelengths $\lambda_{s}$ and $\lambda_{l}$, respectively. To be representative, a surface has to obey a statistically relevant distribution of heights so that, its global mechanical response computed with periodic boundary conditions is equivalent to the response of any statistically similar rough surface. Since the mechanical response of the generated surfaces is affected by their height distributions and the PSD, the surface generation procedure has to include both of them into consideration. While the typical PSD of self-affine surfaces is easily obtained using a random midpoint algorithm [13] or by filtering a white noise [26], the normality of the height distribution of simulated surfaces was, to the best of our knowledge, often ignored.

We will demonstrate that even if available numerical techniques permit to generate a self-affine surface with a small error on the resulting PSD, the distribution of heights strongly depends on the range of wavelengths $\left[\lambda_{s}, \lambda_{l}\right]$ included in the spectrum. In order to quantify the deviation from a Gaussian distribution of heights we introduce the integral $L 2$ error:

$$
\varepsilon(S, N)=\sqrt{\int_{-\infty}^{+\infty}\left|G(h, \bar{h}(S), \sigma(S))-P_{N}^{S}(h)\right|^{2} d h}
$$

where the mean height $\bar{h}(S)$, the standard deviation $\sigma(S)$, and the probability density function (PDF) of heights $P_{N}^{S}$ are extracted from surface $S$ and where 
$N$ is the number of bins used to evaluate the PDF of the surface. The function $G$ stands for the normal distribution

$$
G(h, \bar{h}, \sigma)=\frac{1}{\sqrt{2 \pi} \sigma} \exp \left[\frac{-(h-\bar{h})^{2}}{2 \sigma^{2}}\right]
$$

This measure of the surface representativity has been used in a parametric study to identify the effect of the selected wavelength band $\left[\lambda_{s}, \lambda_{l}\right]$.

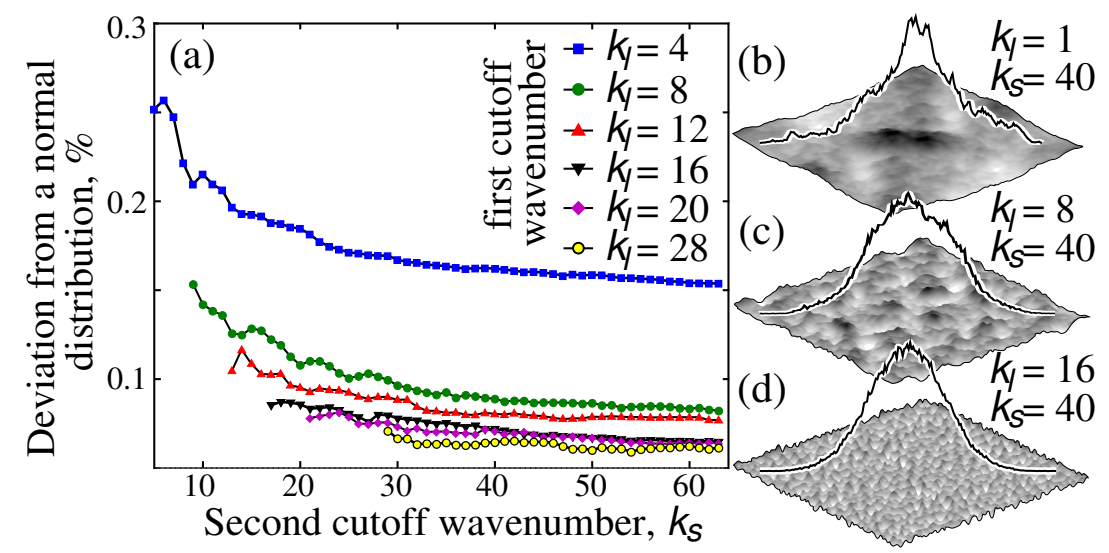

FIG. 5: Mean deviation from the Gaussian distribution (computed over 30 surfaces) with respect to the high cutoff wavenumber $k_{s}$. Various low cutoff wavenumber $k_{l}$ are presented by several curves. The Hurst roughness exponent for all surfaces is $H=0.8$ and the root mean squared height is rms $=0.1$, each surface consists of $1024^{2}$ points.

We have synthesized a large set of self-affine rough surfaces (30 per each combination of $\lambda_{l}$ and $\lambda_{s}$ ) containing $1024 \times 1024$ points by using the filtering technique described in [26]. Fig. 55represents the average error over generated surfaces for Hurst exponent $H=0.8$ and the number of bins $N=500$. It can be observed that the error decreases with increasing $k_{s}$ for all $k_{l}$. However, the value of $k_{l}$ dictates the order of magnitude of the error for all $k_{s}$. Therefore we may assume that for $k_{l}=16$ and $k_{s} \geq 32$ the heights distributions of generated surfaces do not deviate significantly (in average) from the normal one. 


\section{Appendix: contact evolution law}

According to the simulations, the change of the mean contact pressure $\bar{p}=$ $F / A$ with external pressure $p_{0}=F / A_{0}$ decreases approximately as a power law of the real contact area fraction $A / A_{0}$ :

$$
\frac{\partial \bar{p}}{\partial p_{0}}=\beta\left(\frac{A_{0}}{A}\right)^{\mu}, \beta>0,0<\mu<1
$$

In the limit of small contact we suppose the classical linear relation between $A / A_{0}$ and $p_{0}$

$$
\frac{A}{A_{0}}=\frac{\kappa}{\sqrt{|\nabla h|^{2}} E^{*}} p_{0} .
$$

However, in contrast to the Bush-Gibson-Thomas model [5] there is no need to analyze unreachably small fractions of the real contact area. In the limit of the full contact the smoothness of the function is lost

$$
\begin{cases}\frac{\partial \bar{p}}{\partial p_{0}}=\beta, & \text { when } A \rightarrow A_{0} \text { and } A_{0}-A>0, \\ \frac{\partial \bar{p}}{\partial p_{0}}=1, & \text { when } A=A_{0}\end{cases}
$$

In other words the slope of the real contact area is inversely proportional to the contact pressure $p_{0}^{\text {full }}$ needed to establish full contact with the coefficient of proportionality $d\left(A / A_{0}\right) / d p_{0}=(1-\beta) / p_{0}^{\text {full }}$ however, this theory is supposed to be valid only in the range of moderate forces, far from the full contact range. Solving Eq. (4) gives the following law for the evolution of the real contact area

$$
\frac{A}{A_{0}}=\frac{1}{\left[\beta+\left(\frac{\sqrt{|\nabla h|^{2}} E^{*}}{\kappa p_{0}}\right)^{1-\mu}\right]^{1 /(1-\mu)}},
$$

for the considered case we found $\kappa=(1.145 \pm 0.002) \sqrt{2 \pi} \approx 2.870 \pm 0.005$, $\mu=0.55 \pm 0.02, \beta=0.210 \pm 0.005$.

\subsection{Details of the CEL derivation}

Starting with the observation that

$$
\frac{\partial \bar{p}}{\partial p_{0}}=\beta\left(\frac{A_{0}}{A}\right)^{\mu}
$$


Developing the left part gives

$$
\bar{p}=F / A, p_{0}=F / A_{0} \Rightarrow \frac{\partial \bar{p}}{\partial p_{0}}=A_{0} \frac{A-F \partial A / \partial F}{A^{2}}
$$

Substituting this expression in the left part of (66) gives

$$
\frac{A-F \frac{\partial A}{\partial F}}{A^{2}}=\frac{\beta}{A_{0}}\left(\frac{A_{0}}{A}\right)^{\mu}
$$

Grouping terms gives

$$
F \frac{\partial A}{\partial F}=A-\beta A_{0}\left(\frac{A_{0}}{A}\right)^{\mu-2}
$$

Separating variables and integration of both sides reads as

$$
\begin{gathered}
\int_{A_{c}}^{A} \frac{d\left(A / A_{0}\right)}{A / A_{0}-\beta\left(A / A_{0}\right)^{2-\mu}}=\int_{F_{c}}^{F} \frac{d F}{F} \\
\frac{1}{\mu-1} \ln \left(\frac{\left(A / A_{0}\right)^{\mu-1}-\beta}{\left(A_{c} / A_{0}\right)^{\mu-1}-\beta}\right)=\ln \left(F / F_{c}\right)
\end{gathered}
$$

Let $F_{c} \rightarrow 0, A_{c} \rightarrow 0$ and if at very small pressures the real contact area is proportional to the normal force with coefficient $\kappa$, then

$$
A_{c}=\frac{\kappa}{\sqrt{|\nabla h|^{2}} E^{*}} F_{c}
$$

Substituting this limit in the bottom boundary of the left integral in Eq. (8) and integrating, one obtains

$$
\frac{1}{\mu-1} \ln \left(\frac{\left(A / A_{0}\right)^{\mu-1}-\beta}{\left(\frac{\kappa}{\sqrt{|\nabla h|^{2}} E^{*}} F_{c} / A_{0}\right)^{\mu-1}-\beta}\right)=\ln \left(F / F_{c}\right)
$$

Since $\mu-1<0$ and $F_{c}$ tends to zero we can neglect $\beta$ in the denominator

$$
\frac{1}{\mu-1} \ln \left(\frac{\left(A / A_{0}\right)^{\mu-1}-\beta}{\left(\frac{\kappa}{\sqrt{|\nabla h|^{2}} E^{*}} F_{c} / A_{0}\right)^{\mu-1}}\right)=\ln \left(F / F_{c}\right)
$$


Taking the exponential of both parts one gets:

$$
\frac{\left(A / A_{0}\right)^{\mu-1}-\beta}{\left(\frac{\kappa}{\sqrt{|\nabla h|^{2}} E^{*}} F_{c} / A_{0}\right)^{\mu-1}}=\left(F / F_{c}\right)^{\mu-1}
$$

Now we can express the real contact area fraction $A / A_{0}$ as a function of the applied pressure $p_{0}=F / A_{0}$ :

$$
\frac{A}{A_{0}}=\left[\beta+\left(\frac{\kappa p_{0}}{\sqrt{|\nabla h|^{2}} E^{*}}\right)^{\mu-1}\right]^{1 /(\mu-1)}
$$

\section{References}

[1] P. Meakin. Fractals, Scaling and Growth Far from Equilibrium. Cambridge University Press, 1998.

[2] J. Krim and G. Palasantzas. Experimental observations of self-affine scaling and kinetic roughening at sub-micron lengthscales. Int $J \operatorname{Mod}$ Phys B, 9:599-632, 1995.

[3] J. A. Greenwood and J. B. P. Williamson. Contact of nominally flat surfaces. P Roy Soc Lond A Mat, 295:300-319, 1966.

[4] F. P. Bowden and D. Tabor. The Friction and Lubrication of Solids. Oxford University Press, 2001.

[5] A. W. Bush, R. D. Gibson, and T. R. Thomas. The elastic contact of a rough surface. Wear, 35(1):87-111, 1975.

[6] S. Hyun, L. Pei, J. F. Molinari, and M. O. Robbins. Finite-element analysis of contact between elastic self-affine surfaces. Phys Rev E, 70(2):026117, 2004.

[7] C. Campañá and M. H. Müser. Contact mechanics of real vs. randomly rough surfaces: A green's function molecular dynamics study. Europhys Lett, 77:38005, 2007. 
[8] C. Campañá, B. N. J. Persson, and M. H. Müser. Transverse and normal interfacial stiffness of solids with randomly rough surfaces. $J$ PhysCondens Mat, 23:085001, 2011.

[9] A. Almqvist, C. Campañá, N. Prodanov, and B. N. J. Persson. Interfacial separation between elastic solids with randomly rough surfaces: Comparison between theory and numerical techniques. J Mech Phys Solids, 59:2355-2369, 2011.

[10] R. Pohrt and V. L. Popov. Normal contact stiffness of elastic solids with fractal rough surfaces. Phys Rev Lett, 108:104301, 2012.

[11] C. Campañá, M. H. Müser, and M. O. Robbins. Elastic contact between self-affine surfaces: comparison of numerical stress and contact correlation functions with analytic predictions. J Phys-Condens Mat, 20:354013, 2008.

[12] L. Pei, S. Hyun, J. F. Molinari, and M. O. Robbins. Finite element modeling of elasto-plastic contact between rough surfaces. J Mech Phys Solids, 53:2385-2409, 2005.

[13] A. Fournier, D. Fussell, and L. Carpenter. Computer rendering of stochastic models. Commun ACM, 25:371-384, 1982.

[14] V. A. Yastrebov, J. Durand, H. Proudhon, and G. Cailletaud. Rough surface contact analysis by means of the finite element method and of a new reduced model. CR Mecanique, 339:473-490, 2011.

[15] S. Hyun and M. O. Robbins. Elastic contact between rough surfaces: Effect of roughness at large and small wavelengths. Trib Int, 40:14131422, 2007.

[16] C. Putignano, L. Afferrante, G. Carbone, and G. Demelio. The influence of the statistical properties of self-affine surfaces in elastic contacts: A numerical investigation. J Mech Phys Solids, 60:973-982, 2012.

[17] K. L. Johnson. Contact mechanics. Cambridge University Press, 1987.

[18] J. A. Greenwood. A simplified elliptic model of rough surface contact. Wear, 261:191-200, 2006. 
[19] T. R. Thomas. Rough Surfaces. Imperial College Press, second edition, 1999.

[20] M. S. Longuet-Higgins. Statistical properties of an isotropic random surface. Philos T Roy Soc S-A, 250:157-174, 1957.

[21] G. Carbone and F. Bottiglione. Asperity contact theories: Do they predict linearity between contact area and load? J Mech Phys Solids, 56:2555-2572, 2008.

[22] M. Paggi and M. Ciavarella. The coefficient of proportionality k between real contact area and load, with new asperity models. Wear, 268:1020 1029, 2010.

[23] B. N. J. Persson. Theory of rubber friction and contact mechanics. J Chem Phys, 115:3840-3861, 2001.

[24] B. N. J. Persson. Elastoplastic contact between randomly rough surfaces. Phys Rev Lett, 87:116101, 2001.

[25] T. Kanit, S. Forest, I. Galliet, V. Mounoury, and D. Jeulin. Determination of the size of the representative volume element for random composites: statistical and numerical approach. Int J Solids Struct, 40:3647-3679, 2003.

[26] Y. Z. Hu and K. Tonder. Simulation of 3-D random rough surface by 2-D digital filter and fourier analysis. Int J Mach Tool Manu, 32:83-90, 1992.

[27] B. Luan and M. O. Robbins. The breakdown of continuum models for mechanical contacts. Nature, 435:929-932, 2005.

[28] H. M. Stanley and T. Kato. An FFT-based method for rough surface contact. J Tribol-T ASME, 119:481-485, 1997.

[29] The original formulation of the resolution algorithm 28] contains some errors. The most important is the shift of the solution in Fourier space by one wavenumber. A corrected version of this method, validated on many cases, was used for the current study. 